•编者按・

\title{
遥感已经成为生物多样性研究保护与变化监测 不可或缺的技术手段
}

\author{
郭庆华* 刘 瑾 \\ (中国科学院植物研究所植被与环境变化国家重点实验室, 北京 100093)
}

\section{Remote sensing has become an indispensable technology for biodiversity research protection and change monitoring}

Qinghua Guo*, Jin Liu

State Key Laboratory of Vegetation and Environmental Change, Institute of Botany, Chinese Academy of Sciences, Beijing 100093

自1985年美国国家生物多样性论坛第一次筹 备会议提出 “biodiversity”一词至今 (Harper \& Hawksworth, 1995), 生物多样性科学(马克平, 2016) 历经30余年的学科建设发展和保护实践活动, 其维 持生态系统功能、提供生态系统服务、延续人类福 祉的重要性已为大量研究所证实(MA, 2005; Duffy, 2009; Cardinale et al, 2012)。然而, 对于推动《生物 多样性公约》(Convention on Biological Diversity, $\mathrm{CBD}$ )缔约方加强生物多样性监测和保护能力建设、 承担履约责任、实现在《公约》框架下制定的“爱 知生物多样性目标”, 不仅需要强调生物多样性丧 失的严重危害并揭示哪些人类活动是引发生物多 样性危机的驱动因素(Pimm et al, 2014; Celallos et al, 2015), 更需要相关研究从局地、景观、区域、全球 等一系列尺度提供直接反映生物多样性组成及其 变化以及受威胁程度的定性和定量精确描述 (Pasher et al, 2014; 郭庆华等, 2016)。

布庸置疑, 遥感技术是能够提供大规模、长时 间、标准化、全覆盖、高分辨率生物多样性观测信 息的唯一经济可行选择(Turner et al, 2003; Skidmore et al, 2015; Lausch et al, 2015)。无人机、激光雷达、 高光谱、红外相机等新一代遥感技术的出现和不断 完善, 促使生物多样性遥感研究的发展从宏观尺度 到个体尺度, 从平面到立体, 从简单模糊的有/无状
态深入到机理的动态变化监测。但是目前, 学界对 于如何利用快速发展的遥感对地观测技术监测和 量化生物多样性尚未形成普遍共识, 对于确立哪些 遥感监测指标反映特定尺度、某一层次的生物多样 性变化也尚无定论。

2017年11月15日, 中国科学院植物研究所植被 与环境变化国家重点实验室、中国科学院生物多样 性委员会和环境保护部卫星环境应用中心三家单 位联合举办了“第一届生物多样性遥感应用研讨会” 并着手组织本期专辑。通过本专辑有关遥感对地观 测技术应用的最新进展介绍, 希望让读者充分了解: 为什么该技术能够监测并记录生物多样性信息; 涉 及生物多样性实体的遥感定量化过程需要考虑哪 些条件和限制因素; 现有的技术方法可以实现哪些 生物多样性实体信息的定量反演。最终, 希望本专 辑的出版能促使研究人员在应用遥感技术之初就 更多地思考如何建立遥感观测信息与地面实测数 据的紧密联系从而实现二者的互补和有效结合。因 为, 只有当遥感观测信息与地面观测数据密切结合 时, 其在时空覆盖方面的优势和价值才能得以体现, 而且, 地面观测数据往往是校准和评估遥感精度所 必需的。

生物多样性意味着所有来自地球生命体之间 的多样性和差异, 包括陆地、海洋和其他水生生态 
系统以及它们所属的生态复合体, 涵盖了不同组织 层面一从分子、遗传、个体到种群、群落再到生 态系统、景观乃至区域、全球(蒋志刚和马克平, 2009)。但归根结底, 生物多样性的描述和刻画不外 乎从三个维度来进行: 类群组成、结构和功能。相 应地, 生物多样性遥感就是基于不同对地观测平台 和传感器以某种模型方法建立起遥感派生信息与 生物多样性之间的联系, 以此来帮助我们更深刻地 体会我们所在星球惊人的生物多样性, 并能更清晰 地定量理解和认识类群多样性、结构多样性和功能 多样性对人类生存的重要意义。在本专辑中, 郭庆 华等(2018)从观测手段、研究尺度、观测对象和生 物多样性关注点等几方面综述了遥感在生物多样 性研究中的应用现状, 重点分析不同遥感平台的技 术优势和局限性, 并探讨了未来遥感在生物多样性 研究中的应用趋势。文章特别指出: 近地面遥感作 为一个新兴平台, 能够直接观测到物种个体, 正在 将曾被认为不可能实现的植物物种大尺度制图和 分析变成现实。唐志尧等(2018)认为遥感技术已从 传统的光学遥感阶段发展到不同平台、不同维度相 结合的多源遥感阶段, 并逐渐进入以高空间分辨率 和高光谱为特征、以激光雷达为前沿发展方向的综 合遥感阶段。基于此观点, 该文围绕种群动态、种 间关系与群落多样性、功能属性与功能多样性以及 生物多样性保护管理等生物多样性研究热点问题, 系统地论述了航空航天遥感技术在物种多样性研 究与保护领域的应用, 总结了航空航天遥感技术在 研究与物种多样性有关的主要生态学问题中面临 的机遇与挑战。李爱农等(2018)提出开展基于站点 的星空地一体化遥感观测试验, 可以提供物种和生 境的综合定量信息, 与生态模型的有机结合可以刻 画生物多样性的时空格局与动态扩展, 有助于挖掘 过程机理, 提高生物多样性监测的信息化水平, 这 为生物多样性遥感监测提供了新的契机。希望读者 能从这3篇综述文章中全面了解生物多样性遥感的 历史发展、原理方法和前沿动态。

生物多样性遥感的早期应用始于利用卫星遥 感单一数据源形成植被指数估算生物量、生产力, 进行生物多样性预测。由于生物量和生产力遥感模 型的快速发展使得基于 “生产力假说”的植物生物多 样性遥感在全球范围内开展了广泛的应用(Oindo \& Skidmore, 2002)。史林鹭等(2018) 建立了基于
MODIS增强植被指数 (enhanced vegetation index, EVI)的时间序列模型，揭示了鄱阳湖湿地植被从 2000年到2014年植被覆盖和生产力的时空变化趋 势, 发现秋季生长季提前导致生物量过度积累, 降 低了迁飞雁类食源的适口性，需要保证自由连通与 局部水文控制的子湖面积，为越冬候鸟提供更广泛 的食源。随着遥感对地观测平台、传感器种类的日 益丰富，多波段、多极化、多尺度的遥感数据正在 源源不断地产生。从多源融合数据中挖掘新信息成 为植物生物多样性遥感领域内的一个研究热点问 题。本专辑中，王乐等(2018)分时空模式、结构参数、 生理生化参数 3 个方面介绍了利用多源遥感数据监 测红树林的一些重要研究进展，为红树林相关信息 要素提取时如何选择合适的分析方法提供了有力 的参考, 将更好地促进红树林生态系统的生物多样 性保育工作。

相对于植物生物多样性遥感, 动物生物多样性 遥感监测与研究的难度显著增加是不言而喻的。尤 其对于许多珍稀濒危的野生动物来说，其警觉、隐 蔽、夜行的生活习性给涉及种群数量、动态、行为 特征等方面的遥感直接调查取样增添了工作难度。 可喜的是, 本专辑收录的文章有 $1 / 3$ 是有关动物生 物多样性遥感的，这直接反映出遥感技术的发展进 步在逐步克服上述研究困难，推进相关研究工作。 刘雪华等(2018)详细阐述了红外相机数据管理和利 用方面存在的 3 个关键问题，同时以秦岭、卧龙等地 的一些研究为主体列举分析了红外相机照片后续 数据挖掘中 8 个方面的内容。遥感直接获取动物多 样性相关信息仅仅是动物生物多样性研究的一个 方面，而以栖息地质量、空间分布等间接要素为研 究目标的遥感信息获取，对于野生动物保护研究同 等重要。雷倩等(2018)以迁移活动性尤为强烈的岛 类为对象, 综述了可见光遥感、红外遥感、多光谱 遥感、高光谱遥感、激光雷达遥感在获取鸟类活动 及生境信息时涉及行为观察、栖息地选择、生境适 宜性评估和人为干扰探究等不同研究方面时是各 有侧重的，并强调未来的遥感技术在鸟类生物多样 性应用研究中应致力于提供更加明确的光谱信息、 相对简便的解译方法、组合使用方式明晰的数据结 合方式等。物种分布模型是提供生境变化早期预警 并促进及时响应的研究手段, 遥感数据或其衍生产 品是预测物种丰度和分布的重要输入参数。虽然激 
光雷达主动遥感技术已被大量应用于森林三维结 构信息的提取, 但其在物种分布模拟方面的应用研 究还比较缺乏。周中一等(2018)以美国加州内华达 山脉南部地区的食鱼貂(Martes pennanti)的分布模 拟为例，探索LiDAR技术在物种分布模拟中的有效 性, 结果表明LiDAR数据能够提高食鱼貂空间分布 的预测精度, 在物种分布模拟方面的应用潜力亟待 进一步挖掘。

近年来, 得益于无人机产品类型的多元化和成 本降低以及操作软件简单易用, 近地面遥感技术在 生物多样性领域的应用急速发展。一般而言, 该项 技术的应用限制是需要权衡遥感数据可供分析覆 盖的范围和传感器分辨率对于所研究对象的细致 程度(Lausch et al, 2015)。往往厘米级空间分辨率的 传感器对地表覆盖范围较窄, 这对于拼接组装成较 大范围数据集颇具挑战性。而准确的样地坐标位置 是以无人机为代表的近地面遥感平台航摄数据与 地面调查数据融合使用的必要前提。需要充分考虑 制约样地位置测量精度的各种要素并予以足够重 视, 否则可能影响后期的数据融合过程甚至得出错 误的结论。邓云等(2018)通过西双版纳地区10个热 带森林样地及周围区域无人机航摄过程中的地面 控制点测量精度, Photoscan摄影测量软件所得点云 解算精度和照片曝光点重投影精度的对比研究, 建 议在对热带森林进行无人机航摄的过程中必须设 置足够数量、分布相对均匀的地面控制点以控制测 量误差。针对如何在自然保护地应用无人机近地面 遥感技术, 形成无人机和地面相结合的监测技术体 系, 更好地发挥多种调查监测各自的技术优势, 刘 方正等(2018)在梳理了我国不同类型自然保护地生 物多样性保护管理和监测需求的基础上, 归纳提出 了包括图像识别与分类解译、数据反演与格局分 析、数字建模与地表测量、巡护巡检 4 个类别共计 14 个专题的无人机和地面相结合的监测技术方案, 明确了监测时期与频次、监测指标、监测技术的结 合途径以及数据后处理方法等, 为未来自然保护地 精细化管理提供了技术方案储备。

展望未来，遥感技术还在继续不断发展进步， 光谱信息成像化、激光雷达亲民化、生境监测动态 化、空间分析智能化以及模型研究定量化, 都将极 大地提高遥感技术的实时性和运行性，有助于多尺 度、多频率、全天候、高精度和高时效性的生物多
样性遥感监测目标实现。在生物多样性遥感模型建 立过程中如何合理使用遥感数据是非常具有挑战 性的。由于某种程度的时空异质性是所有层级生物 多样性以及影响它们的物理变量所固有的, 因此需 要关注样本大小和遥感与生态数据的特性, 将生境 数据的时空尺度与所关注问题或现象发生的时空 尺度相匹配就尤为重要, 多尺度获取数据的方法是 非常必要的。此外, 需要在遥感对地观测技术体系 中引入数据校准的新概念和框架机制, 以确保在公 众科学日渐盛行、生物多样性监测网络全球重视发 展的大数据时代背景下能够显著提升生物多样性 实体遥感定量分析和预测的能力。希望本期专辑能 从不同的应用视角让读者对生物多样性遥感有一 个崭新的认识。

\section{参考文献}

Cardinale BJ, Duffy JE, Gonzalez A, Hooper DU, Perrings C, Venail P, Narwani A, Mace GM, Tilman D, Wardle DA, Kinzig AP, Daily GC, Loreau M, Grace JB, Larigauderie A, Srivastava DS, Naeem S (2012) Biodiversity loss and its impact on humanity. Nature, 486, 59-67.

Ceballos G, Ehrlich P, Barnosky A, García A, Pringle R, Palmer T (2015) Accelerated modern human-induced species losses: Entering the sixth mass extinction. Science Advances, 1, e1400253.

Deng Y, Wang B, Li Q, Zhang ZM, Deng XB, Cao M, Lin LX (2018) An analysis of lightweight-drone-assisted mapping accuracy in a tropical forest plot. Biodiversity Science, 26, 892-904. (in Chinese with English abstract) [邓云, 王彬, 李强, 张志明, 邓晓保, 曹敏, 林露湘 (2018) 轻小型无 人机航摄技术辅助的热带森林样地测量精度问题探讨. 生物多样性, 26, 892-904.]

Duffy JE (2009) Why biodiversity is important to the functioning of real-world ecosystems. Frontiers in Ecology and the Environment, 7, 437-444.

Guo QH, Hu TY, Jiang YX, Jin SC, Wang R, Guan HC, Yang QL, Li YM, Wu FF, Zhai QP, Liu J, Su YJ (2018) Advances in remote sensing application for biodiversity research. Biodiversity Science, 26, 789-806. (in Chinese with English abstract) [郭庆华, 胡天宇, 姜媛茜, 金时超, 王瑞, 关宏 灿, 杨秋丽, 李玉美, 吴芳芳, 翟秋萍, 刘瑾, 苏艳军 (2018) 遥感在生物多样性研究中的应用进展. 生物多样 性, 26, 789-806.]

Guo QH, Liu J, Li YM, Zhai QP, Wang YC, Wu FF, Hu TY, Wan HW, Liu HM, Shen WM (2016) A near-surface remote sensing platform for biodiversity monitoring: Perspectives and prospects. Biodiversity Science, 24, 1249-1266. (in Chinese with English abstract) [郭庆华, 刘瑾, 李玉美, 翟 秋萍，王永财，吴芳芳，胡天宇，万华伟，刘慧明，申文 
明 (2016) 生物多样性近地面遥感监测: 应用现状与前 景展望. 生物多样性, 24, 1249-1266.]

Haper JL, Hawksworth DL (1995) Preface. In: Biodiversity: Measurement and Estimation (ed. Hawksworth DL), pp. 5-12. Chapman \& Hall, London.

Jiang ZG, Ma KP (2009) Status, challenges and strategy in Conservation Biology. Biodiversity Science, 17, 107-116. (in Chinese with English abstract) [蒋志刚, 马克平 (2009) 保护生物学的现状、挑战和对策. 生物多样性, 17, 107-116.]

Lausch A, Blaschke T, Haase D, Herzog F, Syrbe RU, Tischendorf L, Walz U (2015) Understanding and quantifying landscape structure: A review on relevant process characteristics, data models and landscape metrics. Ecological Modelling, 295, 31-41.

Lei Q, Li JY, Ma KM (2018) Applications of remote sensing technology in avian ecology. Biodiversity Science, 26, 862-877. (in Chinese with English abstract) [雷倩, 李金亚, 马克明 (2018) 遥感技术在鸟类生态学研究中的应用. 生物多样性, 26, 862-877.]

Li AN, Yin GF, Zhang ZJ, Tan JB, Nan X, Ma KP, Guo QH (2018) Space-air-field integrated biodiversity monitoring based on experimental station. Biodiversity Science, 26, 819-827. (in Chinese with English abstract) [李爱农, 尹高 飞, 张正健, 谭剑波, 南希, 马克平, 郭庆华 (2018) 基 于站点的生物多样性星空地一体化遥感监测. 生物多样 性, 26, 819-827.]

Liu FZ, Du JH, Zhou Y, Huang ZP, Li YP, Wang W, Xiao W (2018) Monitoring technology and practice on protected areas biodiversity by integrating unmanned aerial vehicle (UAV) and ground approaches. Biodiversity Science, 26, 905-917. (in Chinese with English abstract) [刘方正, 杜金 鸿, 周越, 黄志旁, 李延鹏, 王伟, 肖文 (2018) 无人机 和地面相结合的自然保护地生物多样性监测技术与实践. 生物多样性, 26, 905-917.]

Liu XH, Wu PF, He XB, Zhao XY (2018) Application and data mining of infra-red camera in the monitoring of species. Biodiversity Science, 26, 850-861. (in Chinese with English abstract) [刘雪华, 武鹏峰, 何祥博, 赵翔宇 (2018) 红外 相机技术在物种监测中的应用及数据挖掘. 生物多样性, 26, 850-861.]

MA (Millennium Ecosystem Assessment) (2005) Ecosystems and Human Well-being: Synthesis. Island Press, Washington, DC.

Ma KP (2016) Hot topics for Biodiversity Science. Biodiversity Science, 24, 1-2. (in Chinese) [马克平 (2016) 生物多样性 科学的热点问题. 生物多样性, 24, 1-2.]

Oindo BO, Skidmore AK (2002) Interannual variability of NDVI and species richness in Kenya. International Journal of Remote Sensing, 23, 285-298.
Pasher J, Smith PA, Forbes MR, Duffea J (2014) Terrestrial ecosystem monitoring in Canada and the greater role for integrated earth observation. Environmental Reviews, 22, 179-187.

Pimm SL, Jenkins CN, Abell R, Brooks TM, Gittleman JL, Joppa LN, Raven PH, Roberts CM, Sexton J (2014) The biodiversity of species and their rates of extinction, distribution, and protection. Science, 344, 987.

Shi LL, Jia YF, Zuo AJ, Ma TH, Lei JL, Lei CG, Wen L (2018) Dynamic change of vegetation cover and productivity of Poyang Lake wetland based on MODIS EVI time series. Biodiversity Science, 26, 828-837. (in Chinese with English abstract) [史林鹭, 贾亦飞, 左奥杰，马童慧，雷佳琳，雷 光春，文力 (2018) 基于MODIS EVI时间序列的鄱阳湖 湿地植被覆盖和生产力的动态变化. 生物多样性, 26 , 828-837.]

Skidmore AK, Pettorelli N, Coops NC, Geller GN, Hansen M, Lucas R, Mucher CA, O'Connor B, Paganini M, Pereira HM, Schaepman ME, Turner W, Wang TJ, Wegmann M (2015) Agree on biodiversity metrics to track from space. Nature, 523, 403-405.

Tang ZY, Jiang MW, Zhang J, Zhang XY (2018) Applications of satellite and air-borne remote sensing in biodiversity research and conservation. Biodiversity Science, 26, 807-818. (in Chinese with English abstract) [唐志尧, 蒋旻炜, 张健, 张新悦 (2018) 航空航天遥感在物种多样性研究与保护 中的应用. 生物多样性, 26, 807-818.]

Turner W, Spector S, Gardiner N, Fladeland M, Sterling E, Steininger M (2003) Remote sensing for biodiversity science and conservation. Trends in Ecology \& Evolution, 18, 306-314.

Wang L, Shi C, Tian JY, Song XN, Jia MM, Li XJ, Liu XM, Zhong RF, Yin DM, Yang SS, Guo XX (2018) Researches on mangrove forest monitoring methods based on multi-source remote sensing. Biodiversity Science, 26, 838-849. (in Chinese with English abstract) [王乐, 时晨, 田金炎，宋晓楠，贾明明，李小娟，刘晓萌，钟若飞，殷 大萌, 杨杉杉, 郭先仙 (2018) 基于多源遥感的红树林监 测. 生物多样性, 26, 838-849.]

Zhou ZY, Liu R, Shi SN, Su YJ, Li WK, Guo QH (2018) Ecological niche modeling with LiDAR data: A case study of modeling the distribution of fisher in the southern Sierra Nevada Mountains, California. Biodiversity Science, 26, 878-891. (in Chinese with English abstract) [周中一, 刘苒, 时书纳, 苏艳军, 李文楷, 郭庆华 (2018) 基于激光雷达 数据的物种分布模拟：以美国加州内华达山脉南部区域 食鱼貂分布模拟为例. 生物多样性, 26, 878-891.]

(责任编辑: 周玉荣) 